\title{
Associação entre o perfil andrológico e a congelação de sêmen de touros da raça Nelore aos dois anos de idade, pré-selecionados pela classificação andrológica por pontos (CAP)
}

\author{
[Relationship between andrologic profile and semen freezing in two-year-old Nelore bulls, \\ pre select by breeding soundness evaluation (BSE)]

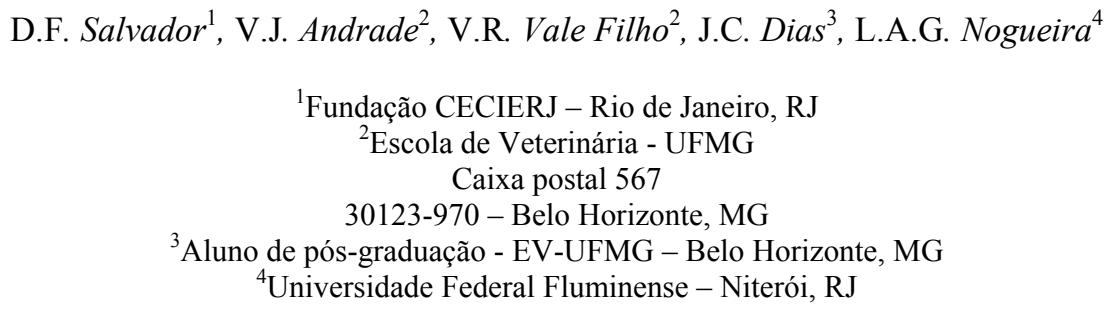

\section{RESUMO}

Avaliaram-se as características andrológicas $(n=163)$ e de congelação do sêmen $(n=55)$ de touros jovens da raça Nelore e estudou-se a associação entre essas avaliações, visando à localização de marcadores para alta fertilidade e congelabilidade do sêmen. Registraram-se diferenças $(\mathrm{P}<0,05)$ no perfil andrológico dos animais entre os anos, com reflexo no sucesso de congelação do sêmen $(\mathrm{P}<0,05)$. Registraram-se 26,6\% e 26,1\% de descarte de ejaculados, respectivamente, pré e pós-congelação, em função dos aspectos físicos do sêmen. As médias gerais de motilidade espermática, vigor e viabilidade pré e pós-congelação foram de $66,4 \pm 5,7 \%$, $4,8 \pm 0,4$ e $76,3 \pm 8,5 \%$ e de $29,4 \pm 8,5 \%, 4,6 \pm 0,6$ e $34,5 \pm 11,1 \%$, respectivamente. A taxa de recuperação póscongelação foi de $44,5 \pm 13,4 \%$. Registraram-se correlações $(\mathrm{P}<0,05)$ entre peso corporal e motilidade $(0,43)$ e padrão de recuperação espermática pós-congelação $(-0,50)$. Correlações significativas $(\mathrm{P}<0,05)$ entre o volume ejaculado e a motilidade espermática $(0,28)$, defeitos espermáticos totais $(-0,32)$ e padrão de recuperação espermática $(-0,37)$ foram estimadas. A correlação entre a concentração espermática e o padrão de recuperação espermática pós-congelação foi de 0,42 . Correlações significativas $(\mathrm{P}<0,05)$ entre o formato dos testículos e a circunferência escrotal $(0,58)$, os defeitos espermáticos totais $(0,33)$ e a área das glândulas vesiculares $(0,43)$ foram observadas também.

Palavras-chave: touro, Nelore, sêmen, andrologia, criopreservação

\begin{abstract}
Andrologic $(n=163)$ and semen freezing $(n=55)$ parameters from young Nelore bulls were evaluated for two consecutive years, as well as their associations searching for andrologic markers for high fertility and semen freezing. Differences in andrologic profiles $(P<0.05)$ between years were registered, reflecting the success of semen freezing, even after andrologic selection. Percentages of 26.6 and 19.5 of discharged ejaculates, pre and post-freezing, respectively, were recorded, due to physical semen parameters. Overall sperm motility, vigor, and viability means were, respectively, $66.4 \pm 5.7 \%, 4.8 \pm 0.4$, and $76.3 \pm 8.5 \%$ pre-freezing; and $29.4 \pm 8.5 \%$, $4.6 \pm 0.6$, and $34.5 \pm 11.1 \%$ post-freezing. The post-freezing recovery mean was $44.5 \pm 13.4 \%$. Significant correlations $(P<0.05)$ between body weight and pre-freezing sperm motility $(0.43)$ and post-freezing sperm recovery standard (-0.50) were estimated. Correlations were also observed $(P<0.05)$ between volume of ejaculate and age (0.50), sperm motility (0.28), total sperm defects (-0.32), and post-freezing sperm recovery standard (-0.37). The correlation between sperm concentration and post-freezing sperm recovery standard was 0.42. Significant correlations $(P<0.05)$ between testes shape and scrotal circumference $(0.58)$, and total sperm defects (0.33) and vesicular gland area (0.43) were also observed.
\end{abstract}

Keywords: bulls, Nelore, semen, andrology, cryopreservation

Recebido em 24 de março de 2007

Aceito em 31 de março de 2008

E-mail: vejoan@dedalus.lcc.ufmg.br 


\section{INTRODUÇÃO}

A seleção andrológica constitui-se na utilização dos exames reprodutivos de rotina de touros para classificação reprodutiva, visando à seleção de características com alta herdabilidade e com reflexos econômicos para a atividade produtiva.

A seleção andrológica tradicional tem se mostrado muitas vezes ineficaz para escolha de touros com alto potencial reprodutivo (Blottner et al., 1990), fato este que tem direcionado as pesquisas na busca de diversos marcadores genéticos entre as características reprodutivas com potencial de quantificação, principalmente molecular e fisiológico (Garner, 1997, Salvador, 2005).

Descobertas de marcadores andrológicos, que expliquem a variabilidade do sucesso na congelação e fertilidade do sêmen de touros, têm sido um forte enfoque nas pesquisas em reprodução animal (Den Daas, 1997, Rodriguez-Martinez, 2004).

A alta variabilidade no desempenho de congelação do sêmen entre os doadores é descrita em várias espécies, sendo os bovinos uma das espécies em que a criopreservação do sêmen apresenta melhor desempenho para utilização em inseminação artificial (Den Daas, 1997; Hafez, 2004, Anchieta et al., 2005).

Vários autores (Kajaestad et al., 1993; Stalhammar et al., 1994; Den Daas, 1997; Roncoletta, 2003) relataram diferenças entre touros, para o sucesso na congelação e a fertilidade do sêmen bovino. Recentes pesquisas em andrologia animal têm sido à busca de marcadores fisiológicos e moleculares, para touros de alto potencial de congelação e fertilidade do sêmen (Roncoletta, 2003, Jobim et al., 2004, Salvador, 2005).

Os objetivos deste trabalho foram avaliar o efeito da seleção andrológica no desempenho da congelação de sêmen e descrever a variabilidade do perfil de congelação de sêmen a campo, buscando-se marcadores fisiológicos para fertilidade e congelabilidade do sêmen em touros jovens da raça Nelore.

\section{MATERIAL E MÉTODOS}

Os experimentos foram realizados com touros jovens (20 a 30 meses) da raça Nelore, pertencentes à Fazenda Mamoneira, município de Unaí-MG, de propriedade da Agropecuária
Naviraí e Mamoneira, em dois anos consecutivos.

Foram examinados no primeiro e segundo anos, respectivamente, 95 e 68 touros Nelore PO, LA e "caras limpas", provenientes de acasalamentos de fêmeas azebuadas com touros Nelore PO de distintas linhagens. Os animais foram criados em condições semi-extensivas, com alimentação a pasto durante os meses de novembro a abril. No período seco (maio a outubro), os animais eram confinados com dieta à base de silagem de capim e ração balanceada. No primeiro ano de exames, os animais permaneceram no regime de confinamento por um menor período, o que refletiu em menor ganho de peso.

As avaliações quanto aos aspectos clínicoandrológicos: circunferência escrotal (CE), biometria testicular (comprimento, largura, volume e forma), glândulas vesiculares (comprimento e largura), aspectos físicos $\mathrm{e}$ morfológicos do sêmen seguiram critérios do CBRA (Manual..., 1998). A classificação andrológica por pontos (CAP) foi realizada de acordo com Vale Filho (1989). Amostras de sêmen foram armazenadas em solução formolsalina para a avaliação da concentração e morfologia espermáticas.

Para aferição mais adequada do perfil de congelação das amostras do sêmen dos touros, as avaliações das viabilidades espermáticas a fresco e pós-congelação foram feitas com o corante eosina-citrato $(0,2 \mathrm{~g}$ de eosina e $0,3 \mathrm{~g}$ de citrato de sódio).

Para cada ano do estudo, do total de touros examinados, com maturidade sexual (aprovados) no momento do primeiro exame andrológico, foram selecionados para a congelação de sêmen (19 no primeiro e 36 no segundo ano) aqueles com maiores pontuações na CAP; CE acima de $30 \mathrm{~cm}$ e motilidade espermática acima de $50 \%$. O sêmen foi coletado com auxílio de eletroejaculador. Foram utilizados apenas aqueles com motilidade superior a $55 \%$, vigor superior a 4 , concentração espermática maior que $400 \times 10^{6} / \mathrm{ml}$ e volume ejaculado maior que $6,0 \mathrm{ml}$. Os critérios para aprovação do sêmen póscongelação foram os recomendados pelo CBRA (Manual..., 1998). O sêmen era considerado aprovado desde que apresentasse motilidade e viabilidade $\geq 30 \%$ e vigor acima de 3 . 
A congelação do sêmen a campo foi realizada de acordo com a metodologia de $\mathrm{ABREU}^{1}$, com a utilização do diluente descrito por Naghase e Niwa, 1964 (11,0\% de lactose, 6,0\% de glicerina, $20 \%$ de gema de ovo e $1 \mathrm{mg} / \mathrm{ml}$ de estreptomicina e $10^{6} \mathrm{UI} / \mathrm{ml}$ de penicilina potássica). Uma alíquota do sêmen, diluída e distribuída em palhetes de $0,5 \mathrm{ml}$, foi mantida por um período de oito horas a $5^{\circ} \mathrm{C}$ para resfriamento e equilíbrio. A congelação foi realizada após a permanência dos palhetes por 15 minutos em vapor de nitrogênio a $-80^{\circ} \mathrm{C}$ e posterior imersão em nitrogênio líquido.

As amostras foram analisadas quanto à motilidade, ao vigor e à viabilidade póscongelação, bem como foram calculados o padrão de recuperação espermática e as taxas de queda de motilidade (TQM) e de viabilidade (TQV).

$\mathrm{Na}$ avaliação dos grupos de touros, os dados foram organizados em delineamento inteiramente ao acaso (DIC) e submetidos à análise de variância. $\mathrm{Na}$ comparação das médias entre grupos, utilizou-se o teste Fisher, segundo Sampaio (1998). Foram estimadas as correlações simples de Pearson entre as variáveis estudadas, utilizando-se os recursos computacionais do pacote estatístico SAS (User's..., 1996).

Para comparação das freqüências de touros aprovados e reprovados andrologicamente e para o congelamento de sêmen, foi utilizada a técnica de qui-quadrado $\left(\chi^{2}\right)$ segundo Sampaio (1998).

\section{RESULTADOS E DISCUSSÃO}

Na Tab. 1 são apresentados os resultados da avaliação reprodutiva dos touros no primeiro e no segundo ano do experimento. Apesar de os animais estarem dentro da mesma faixa etária, os do segundo ano apresentaram maior percentual de aprovados com CAP $>60$ pontos, o que refletiu em maior número de animais selecionados para a congelação de sêmen.

Na Tab. 2, mostram-se as médias dos pesos, circunferências escrotais e características seminais dos touros, no primeiro e no segundo ano do experimento. Apesar da maior média de idade dos touros do primeiro ano, registraram-se diferenças $(\mathrm{P}<0,05)$ no perfil andrológico a favor dos animais do segundo ano.

Os animais do segundo ano do experimento apresentaram-se com maiores $(\mathrm{P}<0,05)$ pesos corporais, motilidade espermática e CAP, ou seja, melhores quadros espermáticos, o que refletiu numa maior porcentagem de animais sexualmente maduros, e, por conseqüência, maior percentual de animais selecionados para os testes de congelação do sêmen.

$\mathrm{Na}$ Tab. 3, são apresentados os resultados da congelação do sêmen realizada a campo, para os touros selecionados pelo perfil andrológico. Foram estabelecidas três diferentes categorias de touros: 1) aprovado = touros que tiveram sêmen congelado e obtiveram aprovação póscongelação; 2) reprovado pós-congelação = touros que tiveram sêmen congelado e foram reprovados na avaliação pós-congelação; 3) reprovado pré- congelação $=$ touros selecionados e que não tiveram sêmen congelado em virtude da baixa qualidade inicial do sêmen.

Tabela 1. Touros jovens da raça Nelore avaliados, classificados e selecionados andrologicamente para a congelação de sêmen, no primeiro e no segundo ano do experimento

\begin{tabular}{llcccc}
\hline \multirow{2}{*}{ Categoria } & \multicolumn{2}{c}{ Classificação } & \multicolumn{2}{c}{ Primeiro Ano } & \multicolumn{2}{c}{ Segundo Ano } \\
\cline { 3 - 6 } & CAP $>60$ pontos & 34 & $35,8 \mathrm{a}$ & 52 & $76,4 \mathrm{~b}$ \\
\multirow{2}{*}{ Aprovados } & $\mathrm{NAP}<60$ pontos & 20 & $21,2 \mathrm{a}$ & 2 & $2,9 \mathrm{~b}$ \\
& Imaturidade Sexual & 30 & $31,5 \mathrm{a}$ & 9 & $13,3 \mathrm{~b}$ \\
\multirow{2}{*}{ Reprovados } & 11 & $11,5 \mathrm{a}$ & 5 & $7,4 \mathrm{~b}$ \\
\hline \multirow{2}{*}{ Total } & Descarte & 95 & 100,0 & 68 & 100,0 \\
Selecionados para congelação & 19 & $20,0 \mathrm{a}$ & 36 & $52,9 \mathrm{~b}$ \\
\hline
\end{tabular}

Letras distintas na linha indicam diferenças pelo teste $\chi^{2}(\mathrm{P}<0,05)$.

${ }^{1}$ ABREU - comunicação pessoal, 2002, Universidade Federal de Minas Gerais, Belo Horizonte, Brasil. 
Tabela 2. Médias de pesos, circunferências escrotais (CE) e características seminais de touros da raça Nelore de dois anos de idade, no primeiro e no segundo ano do experimento

\begin{tabular}{rrrrrrrrr}
\hline Ano & $\mathrm{n}$ & Idade & Peso $(\mathrm{kg})$ & $\mathrm{CE}(\mathrm{cm})$ & Mot (\%) & DM (\%) & DT (\%) & CAP (0100) \\
\hline 1 & 95 & $25,5 \pm 0,7 \mathrm{a}$ & $374,2 \pm 55,0 \mathrm{a}$ & $30,8 \pm 2,6 \mathrm{a}$ & $52 \pm 16 \mathrm{a}$ & $19 \pm 20$ & $34 \pm 24$ & $53 \pm 19 \mathrm{a}$ \\
2 & 68 & $23,7 \pm 2,0 \mathrm{~b}$ & $410,9 \pm 46,2 \mathrm{~b}$ & $31,1 \pm 2,2 \mathrm{a}$ & $64 \pm 10 \mathrm{~b}$ & $16 \pm 16$ & $25 \pm 19$ & $69 \pm 17 \mathrm{~b}$
\end{tabular}

Mot = motilidade; CAP = classificação andrológica por pontos; $\mathrm{DM}=$ defeitos maiores; $\mathrm{DT}=$ defeitos totais.

Letras distintas na coluna indicam diferença entre valores, pelo teste Fisher $(\mathrm{P}<0,05)$.

As variáveis defeitos maiores e totais não foram comparadas.

Tabela 3. Número e percentual de touros selecionados andrologicamente, por ano, de acordo com a classificação de congelação de sêmen

\begin{tabular}{ccccc}
\hline \multirow{2}{*}{ Classificação } & \multicolumn{2}{c}{ Primeiro ano } & \multicolumn{2}{c}{ Segundo ano } \\
\cline { 2 - 5 } & Número & $\%$ & Número & $\%$ \\
\hline Aprovado & 9 & $47,4 \mathrm{a}$ & 17 & $47,2 \mathrm{a}$ \\
Reprovado pós-congelação & 2 & $10,5 \mathrm{a}$ & 15 & $41,1 \mathrm{~b}$ \\
Reprovado pré-congelação & 8 & $42,1 \mathrm{a}$ & 4 & $11,1 \mathrm{~b}$ \\
Total & 19 & 100,0 & 36 & 100,0 \\
\hline
\end{tabular}

Letras distintas na linha indicam diferença entre valores, pelo teste $\chi^{2}(\mathrm{P}<0,05)$.

Dos animais selecionados no primeiro e no segundo ano do experimento, o percentual de animais aprovados no congelamento no segundo foi semelhante ao do primeiro ano, apesar do melhor quadro seminal apresentado na Tab. 2, mesmo após a seleção andrológica. Este resultado mostra que a seleção andrológica pode propiciar a escolha de reprodutores superiores, mas por si só não é capaz de predizer eficazmente o potencial de congelação do sêmen em touros jovens da raça Nelore.

Para as duas avaliações consecutivas, houve descarte médio de $26,6 \%$, em função de aspectos físicos do sêmen (reprovado pré-congelação), resultado semelhante aos obtidos por Anchieta et al. (2005), que avaliaram 19.868 ejaculações de 394 touros e registraram variações de 23,0 a $29,0 \%$ de descarte de ejaculados pelo mesmo motivo, com variações entre as estações climáticas. Já com base nos critérios adotados para o processo da congelação do sêmen (reprovação pós-congelação), registrou-se média 26,1\%, para os dois anos. Este resultado foi maior que a média observada por Anchieta et al. (2005).

As médias gerais de motilidade espermática, vigor e viabilidade (vivo-mortos) pré-congelação foram $66,4 \pm 5,7 \%, \quad 4,8 \pm 0,4 \%$ e $76,3 \pm 8,5 \%$, respectivamente. Estes resultados foram superiores aos registrados por Anchieta et al. (2005). As médias pós-congelação, para as mesmas características foram, respectivamente, de $29,4 \pm 8,5 \%, \quad 4,6 \pm 0,6 \%$ e $34,5 \pm 11,1 \%$, ligeiramente menores que os resultados de Anchieta et al. (2005), possivelmente em razão da utilização da metodologia de congelação do sêmen a campo, na qual há maiores perdas espermáticas do que nas centrais de processamento de sêmen.

Na Tab. 4, são apresentadas as médias de pesos corporais, $\mathrm{CE}$ e características seminais do grupo de touros aprovados e reprovados para $\mathrm{o}$ congelação do sêmen a campo.

Observaram-se diferenças $(\mathrm{P}<0,05)$ apenas para motilidade espermática entre os touros aprovados e reprovados para o congelação de sêmen. Estes resultados mostram que, após a seleção andrológica prévia pela $\mathrm{CAP}$, as características de peso, $\mathrm{CE}$ e aspectos morfológicos do sêmen têm baixa influência no sucesso da congelação de sêmen de touros jovens da raça Nelore. A motilidade inicial do sêmen antes da congelação é importante para os melhores resultados póscongelação. 


\section{Associação entre o perfil andrológico...}

Tabela 4. Médias de pesos, circunferências escrotais (CE) e características seminais de touros da raça Nelore aprovados e reprovados na classificação de congelação do sêmen

\begin{tabular}{ccccccccc}
\hline Classificação & $\mathrm{N}$ & Idade (m) & Peso (kg) & CE (cm) & Mot (\%) & DM (\%) & DT (\%) & CAP \\
\hline Aprovado & 26 & $24,2 \pm 1,7 \mathrm{a}$ & $391,7 \pm 30,7 \mathrm{a}$ & $31,7 \pm 1, \mathrm{a}$ & $66 \pm 6 \mathrm{a}$ & $8 \pm 6$ & $18 \pm 7$ & $75 \pm 13 \mathrm{a}$ \\
Reprovado & 29 & $25,2 \pm 2,3 \mathrm{a}$ & $403,1 \pm 62,6 \mathrm{a}$ & $32,1 \pm 1, \mathrm{a}$ & $62 \pm 8 \mathrm{~b}$ & $11 \pm 4$ & $19 \pm 6$ & $69 \pm 11 \mathrm{a}$
\end{tabular}

Letras distintas na coluna indicam diferença entre valores, pelo teste Fisher $(\mathrm{P}<0,05)$.

Mot = motilidade; $\mathrm{CE}=$ circunferência escrotal; $\mathrm{DM}=$ defeitos maiores; DT = defeitos totais; $\mathrm{CAP}=\mathrm{Classificação}$ andrológica por pontos (0-100). As variáveis defeitos maiores e totais não foram comparadas.

Com as medidas representativas da qualidade da congelação (motilidade e viabilidade pré e póscongelação), efetuaram-se os cálculos de TQM e $\mathrm{TQV}$, para os 43 touros cujo sêmen foi congelado, que foram, respectivamente, de $37,0 \pm 9,7 \%$ e de $41,9 \pm 10,8 \%$.

Outra medida importante de avaliação da congelação de sêmen em bovinos é a taxa de recuperação pós-congelação, sugerida por Hafez (2004). A taxa de recuperação pós-congelação foi de $44,5 \pm 13,4 \%$.

Na Tab. 5, são apresentados os resultados das principais medidas usadas para avaliação da congelação do sêmen, entre os touros aprovados e reprovados pós-congelação.

As características de motilidade pré-congelação, volume ejaculado, concentração espermática, TQM, TQV e taxa de recuperação espermática pós-congelação do sêmen foram diferentes entre os grupos $(\mathrm{P}<0,05)$. Apesar de a viabilidade estar altamente associada à motilidade espermática, não houve diferença entre os grupos para a viabilidade espermática pré-congelação $(\mathrm{P}>0,05)$.

Tabela 5. Médias das características seminais e de congelação de sêmen de touros da raça Nelore de dois anos de idade (aprovados e reprovados após a congelação do sêmen)

\begin{tabular}{cccccccc} 
Classificação & N & VPC (\%) & VE (ml) & TQV (\%) & TQM (\%) & TR (\%) & CES x $10^{6}$ \\
\hline Aprovados & 26 & $77 \pm 9 \mathrm{a}$ & $10,1 \pm 2,5 \mathrm{a}$ & $37 \pm 10 \mathrm{a}$ & $31 \pm 7 \mathrm{a}$ & $53 \pm 8 \mathrm{a}$ & $462 \pm 190 \mathrm{a}$ \\
Reprovados & 17 & $76 \pm 8 \mathrm{a}$ & $11,7 \pm 2,2 \mathrm{~b}$ & $47 \pm 6 \mathrm{~b}$ & $46 \pm 6 \mathrm{~b}$ & $31 \pm 7 \mathrm{~b}$ & $301 \pm 191 \mathrm{~b}$
\end{tabular}

$\mathrm{VPC}=$ viabilidade pré-congelação; $\mathrm{VE}=$ volume do ejaculado; $\mathrm{TQV}=$ taxa de queda de viabilidade; $\mathrm{TQM}=$ taxa de queda de motilidade; TR = taxa de recuperação espermática; $\mathrm{CES}=$ concentração espermática.

Letras distintas na coluna indicam diferença entre valores, pelo teste Fisher $(\mathrm{P}<0,05)$.
Os resultados das diferenças para a concentração espermática e o volume ejaculado indicam que ejaculados com alta concentração espermática e menor volume ejaculado, na coleta completa por eletroejaculação, podem estar associados ao melhor desempenho de touros na congelação.

Diante dos resultados apresentados, observa-se que houve grande variabilidade nos resultados da congelação do sêmen de touros jovens da raça Nelore, mesmo após a seleção andrológica pela CAP.

$\mathrm{Na}$ Tab. 6, são apresentadas as correlações entre os dados de idade, peso, biometria testicular, características seminais e desempenho para congelação do sêmen dos touros.

A idade dos animais correlacionou-se positivamente $(\mathrm{P}<0,05) \quad$ com a viabilidade espermática, os defeitos espermáticos totais, o volume ejaculado e o tamanho das glândulas vesiculares com valores de 0,31, 0,50 e 0,30, respectivamente. 
Tabela 6. Correlações e graus de significância entre características andrológicas e de congelação de sêmen de touros da raça Nelore de dois anos de idade

\begin{tabular}{|c|c|c|c|c|c|c|c|c|}
\hline & Idade (m) & Peso (kg) & $\mathrm{CE}(\mathrm{cm})$ & Mot (\%) & VE (\%) & Vol (ml) & CES & Forma \\
\hline Peso & $\begin{array}{l}0,43 \\
0,01\end{array}$ & - & - & - & - & - & - & \\
\hline $\mathrm{CE}$ & $\begin{array}{c}0,41 \\
0,005\end{array}$ & $\begin{array}{l}0,12 \\
0,50\end{array}$ & - & - & - & - & - & \\
\hline Mot & $\begin{array}{c}-0,08 \\
0,58\end{array}$ & $\begin{array}{l}0,43 \\
0,01\end{array}$ & $\begin{array}{c}-0,02 \\
0,85\end{array}$ & - & - & - & - & \\
\hline VE (\%) & $\begin{array}{l}0,31 \\
0,03\end{array}$ & $\begin{array}{c}-0,02 \\
0,88\end{array}$ & $\begin{array}{c}-0,13 \\
0,37\end{array}$ & $\begin{array}{l}0,19 \\
0,20\end{array}$ & - & - & - & \\
\hline Vol (ml) & $\begin{array}{c}0,50 \\
0,006\end{array}$ & $\begin{array}{l}0,09 \\
0,62\end{array}$ & $\begin{array}{c}-0,13 \\
0,37\end{array}$ & $\begin{array}{l}0,28 \\
0,05\end{array}$ & $\begin{array}{c}-0,05 \\
0,74\end{array}$ & - & - & \\
\hline CES & $\begin{array}{l}0,06 \\
0,69\end{array}$ & $\begin{array}{c}-0,23 \\
0,19\end{array}$ & $\begin{array}{l}0,10 \\
0,51\end{array}$ & $\begin{array}{c}-0,06 \\
0,68\end{array}$ & $\begin{array}{c}-0,08 \\
0,57\end{array}$ & $\begin{array}{c}-0,12 \\
0,41\end{array}$ & - & \\
\hline Forma & $\begin{array}{l}0,07 \\
0,62\end{array}$ & $\begin{array}{c}-0,12 \\
0,50\end{array}$ & $\begin{array}{c}0,58 \\
0,0001\end{array}$ & $\begin{array}{c}-0,19 \\
0,21\end{array}$ & $\begin{array}{c}-0,13 \\
0,39\end{array}$ & $\begin{array}{l}0,08 \\
0,59\end{array}$ & $\begin{array}{l}0,09 \\
0,57\end{array}$ & 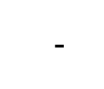 \\
\hline VES & $\begin{array}{l}0,30 \\
0,04\end{array}$ & $\begin{array}{c}-0,02 \\
0,87\end{array}$ & $\begin{array}{c}0,50 \\
0,0005\end{array}$ & $\begin{array}{l}0,06 \\
0,69\end{array}$ & $\begin{array}{c}-0,07 \\
0,61\end{array}$ & $\begin{array}{r}-0,00 \\
0,96\end{array}$ & $\begin{array}{l}0,14 \\
0,35\end{array}$ & $\begin{array}{c}0,43 \\
0,004\end{array}$ \\
\hline P.rec (\%) & $\begin{array}{l}0,04 \\
0,79\end{array}$ & $\begin{array}{l}-0,50 \\
0,003\end{array}$ & $\begin{array}{c}-0,06 \\
0,68\end{array}$ & $\begin{array}{c}-0,20 \\
0,19\end{array}$ & $\begin{array}{l}0,16 \\
0,29\end{array}$ & $\begin{array}{c}-0,37 \\
0,01\end{array}$ & $\begin{array}{c}0,42 \\
0,004\end{array}$ & $\begin{array}{c}-0,06 \\
0,70\end{array}$ \\
\hline
\end{tabular}

$\overline{\mathrm{CE}}=$ circunferência escrotal; Mot $=$ motilidade; $\mathrm{DM}=$ defeitos maiores; $\mathrm{VE}=$ viabilidade; Vol $=$ volume; $\mathrm{DT}=$ defeitos totais; CES = concentração espermática; P. rec = padrão de recuperação do sêmen pós-congelação; VES= área das glândulas vesiculares; Forma = relação do formato dos testículos (largura/comprimento), onde maior valor numérico representa tendência ao formato oval.

Observaram-se correlações significativas entre a forma dos testículos e a CE $(0,58)$ e a área das glândulas vesiculares $(0,43)$.

Houve correlação positiva entre o peso corporal dos animais e a motilidade espermática précongelação $(0,43)$, e negativa $(-0,50, \mathrm{P}<0,05)$ com o padrão de recuperação espermática póscongelação, ou seja, quanto maior o peso corporal dos touros, maior a motilidade espermática inicial e menor o desempenho na congelação do sêmen.

Observou-se correlação entre a motilidade espermática inicial e a taxa de queda de motilidade do sêmen $(0,48, \mathrm{P}<0,05) \mathrm{e}$, em menor intensidade, com o padrão de recuperação espermática pós-congelação $(-0,20, \quad \mathrm{P}>0,05)$. Estes resultados, aparentemente contraditórios, indicam que touros com maior motilidade espermática inicial têm maior percentual de queda da motilidade e, por conseguinte, menor taxa de recuperação espermática, ou seja, a maior motilidade inicial não garante, por si só, bom desempenho na congelação, porém permite que animais com altas taxas de queda da motilidade ainda apresentem aprovação no sêmen póscongelação.

Houve correlação positiva $(\mathrm{P}<0,05)$ entre volume do ejaculado coletado e idade $(0,50)$ e motilidade espermática $(0,28)$. Observou-se correlação negativa entre volume ejaculado e padrão de recuperação espermática pós-congelação $(-0,37)$.

Estes resultados diferem dos resultados obtidos por Salvador, 2001 e Dias et al., 2006, que, em avaliações andrológicas de rotina, não registraram correlações significativas entre $o$ volume ejaculado e os outros parâmetros do sêmen, a não ser com a área das glândulas vesiculares. Isto se deve, provavelmente, ao fato de que, neste experimento, foi realizada a coleta completa do ejaculado dos touros, buscando-se o 


\section{Associação entre o perfil andrológico...}

esgotamento dos animais em coletas seqüenciadas, sendo coletados e avaliados todos os estádios da ejaculação que apresentavam espermatozóides.

Observou-se correlação positiva entre a concentração espermática e o padrão de recuperação espermática pós-congelação $(0,42$, $\mathrm{P}<0,05$ ), sugerindo que touros jovens com maior concentração espermática no ejaculado têm tendência a melhor desempenho para congelação do sêmen.

\section{CONCLUSÕES}

Em touros jovens da raça Nelore, a seleção andrológica tradicional é insuficiente para prever o desempenho na congelação do sêmen, porém o peso corporal, a idade, o formato dos testículos, a concentração espermática, o volume ejaculado completo, e a motilidade espermática influenciam na congelação. Recomenda-se a utilização de testes de congelação do sêmen na fazenda, para touros jovens selecionados pela CAP, como critério seletivo de animais com alto potencial genético para serem utilizados em centrais de produção e processamento de sêmen.

\section{REFERÊNCIAS BIBLIOGRÁFICAS}

ANCHIETA, M.C.; VALE FILHO, V.R.; COLOSIMO, E. et al. Descarte e congelabilidade do sêmen de touros de raças zebuínas e taurinas em central de inseminação artificial no Brasil. Arq. Bras. Med. Vet. Zootec., v.57, p.196-204, 2005.

BLOTTNER, S.; NEHRING, H.; TORNER, H. Individual diferences in capacitation of bull spermatozoa by heparin in vitro: relationship to fertility. Theriogenology, v.34, n.3, p.619-628, 1990.

DEN DAAS, J.H.G. Prediction of bovine Male Fertility. 1997. 168f. Thesis (Doctor of Animal Science). Landbouwuniversiteit te wangeningen, Alemanha.

DIAS, J.C.; ANDRADE, V.J.; FRIDRICH, A.B. Estimativas de parâmetros genéticos de características reprodutivas de touros Nelore, de dois e três anos de idade. Arq. Bras. Med. Vet. Zootec., v.58, p.388-393, 2006.

GARNER, D.L. Ancillary tests of bull semen quality. Vet. Clin. N. Amn.: Food animal practice. v.13, p.313-330, 1997.

HAFEZ, E.S.E. Reprodução Animal. 7.ed. São Paulo: Manole, 2004. 513p.
JOBIM, M.I.M., OBERST, E.R., SALBEGO, C.G. et al. Two-dimensional polyacrylamide gel electrophoresis of bovine seminal plasma proteins and their relation with semen freezability. Theriogenology. v.61, p.255-266, 2004.

KJAESTAD, H.; ROPSTAD, E.; ANDERSEN, K.B. Evaluation of spermatological parameters used to predict the fertility of frozen bull semen. Acta Vet. Scand., v.34, p.299-303, 1993.

MANUAL para exame andrológico e avaliação de sêmen animal. 2.ed. Belo Horizonte: CBRA, 1998.

NAGASE, H; NIWA, T. Deep freezing bull semen in concentrated pellet form. I. Factors affecting survival of spermatozoa. In: INTERNATIONAL CONGRESS ON ANIMAL REPRODUTION AND ARTIFICIAL INSEMINATION, 5., 1964, Trento. Proc... Trento, 1964, p.410-415.

RODRIGUEZ-MARTINEZ, H. Selection of bull spermatozoa by swim-up. In: INTERNATIONAL CONGRESS ON ANIMAL REPRODUCTION, 15., 2004, Porto Seguro. Anais... Porto Seguro, 2004. p.85. (Workshops communications)

RONCOLETTA, M. Perfil bidimensional de proteinas de membrana de espermatozóides e plasma seminal, relacionados com a fertilidade e com a congelabilidade do sêmen de touros. 2003. 104f. Tese (Doutorado) Faculdade de Ciências Agrárias e Veterinárias, UNESP, Jaboticabal.

SALVADOR. D.F. Perfis andrológico, de comportamento sexual e desempenho reprodutivo de touros Nelore desafiados com fêmeas em estro sincronizado. 2001. 53f. Dissertação (Mestrado) - Escola de Veterinária, Universidade Federal de Minas Gerais, Belo Horizonte.

SALVADOR. D.F. Perfis cromatográficos e eletroforéticos de proteinas com afinidade a heparina do sêmen de touros jovens da raça Nelore e suas associações com a seleção andrológica, congelação do sêmen e reação acrossômica induzida (RAI). 2005. 57f. Tese (Doutorado) - Escola de Veterinária, Universidade Federal de Minas Gerais, Belo Horizonte.

SAMPAIO, I.B.M. Estatística aplicada à experimentação animal. Belo Horizonte: FEP-MVZ, 1998. 221p.

STALHAMMAR, E.M.; JANSON, L.; PHILIPSSON, J. The impact of semen motility on non-return rate in preselected dairy bulls. Reprod. Nutr. Dev., v.34, p.37-45, 1994.

USER'S guide. Cary, NC: SAS Institute Inc, 1996.

VALE FILHO, V.R. Padrões de sêmen bovino para o Brasil; análise e sugestões. In: CONGRESSO BRASILEIRO REPRODUÇÃO ANIMAL, 8., 1989, Belo Horizonte. Anais... Belo Horizonte: CBRA, 1989. p.94118 (Palestra). 